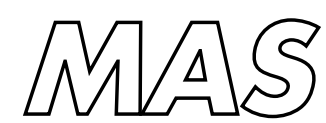

Modelling, Analysis and Simulation

Modelling, Analysis and Simulation
MAS Pattern forming pulled fronts: bounds and universal
convergence

Ute Ebert, Willem Spruijt, Wim van Saarloos

Report MAS-E0318 December 9, 2003 
CWI is the National Research Institute for Mathematics and Computer Science. It is sponsored by the Netherlands Organization for Scientific Research (NWO).

CWI is a founding member of ERCIM, the European Research Consortium for Informatics and Mathematics.

CWI's research has a theme-oriented structure and is grouped into four clusters. Listed below are the names of the clusters and in parentheses their acronyms.

Probability, Networks and Algorithms (PNA)

Software Engineering (SEN)

Modelling, Analysis and Simulation (MAS)

Information Systems (INS)

Copyright (C) 2003, Stichting Centrum voor Wiskunde en Informatica

P.O. Box 94079, 1090 GB Amsterdam (NL)

Kruislaan 413, 1098 SJ Amsterdam (NL)

Telephone +31205929333

Telefax +31205924199

ISSN 1386-3703 


\title{
Pattern forming pulled fronts: bounds and universal convergence
}

\begin{abstract}
We analyze the dynamics of pattern forming fronts which propagate into an unstable state, and whose dynamics is of the pulled type, so that their asymptotic speed is equal to the linear spreading speed $v^{*}$. We discuss a method that allows to derive bounds on the front velocity, and which hence can be used to prove for, among others, the Swift-Hohenberg equation, the Extended Fisher-Kolmogorov equation and the cubic Complex Ginzburg-Landau equation, that the dynamically relevant fronts are of the pulled type. In addition, we generalize the derivation of the universal power law convergence of the dynamics of uniformly translating pulled fronts to both coherent and incoherent pattern forming fronts. The analysis is based on a matching analysis of the dynamics in the leading edge of the front, to the behavior imposed by the nonlinear region behind it. Numerical simulations of fronts in the Swift-Hohenberg equation are in full accord with our analytical predictions.
\end{abstract}

2000 Mathematics Subject Classification: 35G25, 37L10

Keywords and Phrases: pulled fronts, pattern formation, algebraic convergence, Swift-Hohenberg-eq., Cahn-Hilliard-eq., complex Ginzburg-Landau-eq. 


\title{
Pattern forming pulled fronts: bounds and universal convergence
}

\author{
Ute Ebert $^{1,2}$, Willem Spruijt ${ }^{3}$ and Wim van Saarloos ${ }^{3}$ \\ 1 CWI, Postbus 94079, 1090 GB Amsterdam, The Netherlands, \\ 2 Department of Physics, TU Eindhoven, Postbus 513, 5600 MB Eindhoven, \\ The Netherlands, \\ ${ }^{3}$ Instituut-Lorentz, Leiden University, Postbus 9506, 2300 RA Leiden, \\ The Netherlands
}

\begin{abstract}
We analyze the dynamics of pattern forming fronts which propagate into an unstable state, and whose dynamics is of the pulled type, so that their asymptotic speed is equal to the linear spreading speed $v^{*}$. We discuss a method that allows to derive bounds on the front velocity, and which hence can be used to prove for, among others, the Swift-Hohenberg equation, the Extended Fisher-Kolmogorov equation and the cubic Complex Ginzburg-Landau equation, that the dynamically relevant fronts are of the pulled type. In addition, we generalize the derivation of the universal power law convergence of the dynamics of uniformly translating pulled fronts to both coherent and incoherent pattern forming fronts. The analysis is based on a matching analysis of the dynamics in the leading edge of the front, to the behavior imposed by the nonlinear region behind it. Numerical simulations of fronts in the Swift-Hohenberg equation are in full accord with our analytical predictions.
\end{abstract}

\section{Introduction}

In the last few years, it has become clear that when considering a problem of a front which propagates into an unstable state, it is crucial to distinguish two different classes, according to whether their asymptotic speed is equal to or larger than the linear spreading speed $v^{*}$. The linear spreading speed is a simple concept that dates back to developments in plasma physics and fluid dynamics that took place almost half a century ago $[1,2,3,4]$. It is the asymptotic speed with which an initially localized perturbation about the unstable state spreads into this unstable state according to the linear dynamics, the dynamics obtained by linearizing the dynamical equations about the unstable state. For any deterministic dynamical equation this linear spreading speed $v^{*}$ 
can be determined explicitly from a long-time asymptotic saddle-point type analysis of the Green's function of the relevant dynamical equation. In practice, therefore, $v^{*}$ is given explicitly by the dispersion relation of Fourier modes obeying the linearized dynamical equation $[1,2,3,4,5,6,7]$.

Given the existence of a finite linear spreading speed $v^{*}$ for a given problem, only two different types of asymptotic front solutions can emerge starting from "steep" or "sufficiently localized" initial conditions: either the asymptotic velocity of the nonlinear front is equal to $v^{*}$ or it is larger than $v^{*}$. In the first case we speak of "pulled fronts", as such fronts are essentially being pulled along by the growth and spreading of the linear dynamcs in the leading edge where the linearized dynamical equations can be used. In the second case of fronts whose asymptotic speed is larger than $v^{*}$, we speak of pushed fronts $[5,6,7,8,9]$. Because the essential dynamics of pulled fronts is actually taking place in the region ahead of the nonlinear front region, their properties are very different from pushed fronts or other fronts, domain walls or kink solutions whose properties are determined by a nonlinear eigenvalue problem: the singular perturbation theory which is normally used to map weakly curved fronts onto a moving boundary problem, breaks down for pulled fronts [10], and their velocity and shape converge with universal power laws to their asymptotic value and shape. For nonlinear diffusion equations of the type studied by Fisher [11] and Kolmogorov et al. [12] the first term expressing this power law convergence was already derived in 1983 by Bramson [13], but we have recently found that this slow power law convergence can be summarized in one single exact equation that governs any pulled front which converges to a uniformly translating solution $[5,6,14]$. For a review of many of these results, see [7].

As it turns out, the matching analysis on which the derivation of the power law convergence is based (see also [15]), requires only minimal input on the form of the nonlinear uniformly translating pulled front solution to which the front solution converges - the explicit expressions for the velocity convergence are all obtained from a proper Ansatz for the asymptotic expansion of the front solutions in the leading edge, the region where the dynamical equation can be linearized. It is the purpose of this paper to show that this part of the analysis can be easily generalized to dynamical equations whose dynamics is pattern forming, i.e., whose asymptotic front solutions are not uniformly translating. An example of such a front in the Swift-Hohenberg equation is shown in Fig. 1. This conclusion was already announced without derivation in [16]. In fact, the asymptotic relaxation formula which we derive here also applies to incoherent pattern forming fronts - the reason is that the linear spreading dynamics is always coherent, irrespective of whether the dynamics in the nonlinear region behind the front is coherent or incoherent $[7,16]$. While the asymptotic expansion in the leading edge which we will discuss here thus pertains to both types of fronts, we shall focus our discussion of the application of the formula on coherent pattern forming fronts. 


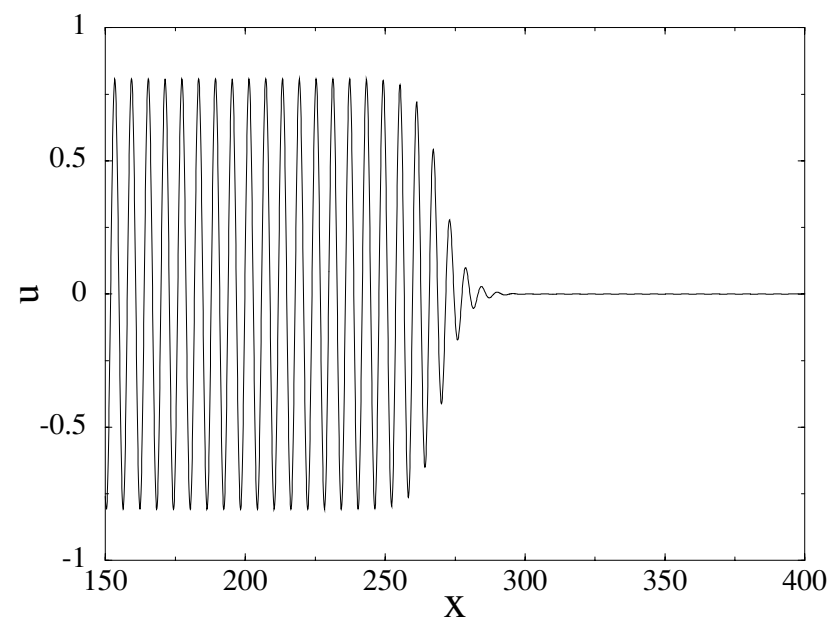

Fig. 1. Snapshot of a front in the Swift-Hohenberg equation (10) for $\varepsilon=0.5$. The front propagates to the right into the region where $u$ is in the unstable state $u=0$.

One of the simplest examples of a dynamical equation whose pattern forming fronts are coherent is the Swift-Hohenberg equation, and we will therefore use this equation to illustrate and test our analytical results. In fact, the SwiftHohenberg equation has often played a role in studies of front propagation $[17,18,19,20,21,22]$ - it is essentially the only equation with pattern forming fronts for which a number of exact results (including the convergence to a pulled front solution) are known [19,20,21,22].

Because there are so few rigorous results for pattern forming fronts in general, we will, before turning to the analysis of the front convergence, discuss a method which allows us to derive a bound on the velocity for pattern forming fonts, like the Swift-Hohenberg equation, the Extended Fisher-Kolmogorov equation, or the cubic Complex Ginzburg-Landau equation. Although our argument is in essence a simplified version of the line of analysis Collet and Eckmann [22] use to prove that fronts in the Swift-Hohenberg equation are pulled, we do want to show the reader how in just a few lines one can prove that fronts in pattern forming equations are pulled: we think that the method holds the promise for many new rigorous results on front propagation.

The layout of this paper is as follows. In the next section we first discuss our method to derive a bound on the front velocity. Then, in section III we perform the asymptotic expansion of the dynamics of the leading edge of a pattern forming front, which gives the expressions for the convergence of the front velocity and shape to their asymptotic behavior. In section IV we illustrate these results with numerical solutions of the Swift-Hohenberg equation, and we close the paper with a brief summary. 


\section{The linear spreading velocity as a rigorous upper bound}

\subsection{The linear spreading velocity}

We consider a generic dynamical equation for some generic dynamical variable $\phi$, whose stationary state $\phi=0$ is linearly unstable, and whose dispersion relation is given by $\omega(k)$. This means that a Fourier perturbation $e^{i k x}$ of the unstable state evolves under the linear dynamics as $e^{-i \omega(k) t+i k x}$. Associated with the linear dynamical problem is a linear spreading velocity $v^{*}$, the velocity with which an initially localized perturbation spreads asymptotically into the unstable state according to the linearized dynamics. The asymptotic spreading is simply determined by a long-time saddle point analysis of the Green's function of the linear equation. From this analysis, one finds $v^{*}$ explicitly in terms of $\omega(k)$ as $[1,2,3,4,5,6,7]$

$$
\left.\frac{\mathrm{d} \omega(k)}{\mathrm{d} k}\right|_{k^{*}}=\frac{\operatorname{Im} \omega\left(k^{*}\right)}{\lambda^{*}}, \quad v^{*}=\frac{\operatorname{Im} \omega\left(k^{*}\right)}{\lambda^{*}}, \quad k^{*} \equiv q^{*}+i \lambda^{*} .
$$

The first equation determines the saddle point value $k^{*}$ in the complex plane, and the second one then gives the linear spreading velocity $v^{*}$. The third equation fixes our notation for the splitting of $k^{*}$ into real part $q^{*}$ and imaginary part $\lambda^{*}$ for the remainder of the paper. The complex parameter

$$
\left.\mathcal{D} \equiv \frac{i}{2} \frac{\mathrm{d}^{2} \omega(k)}{\mathrm{d} k^{2}}\right|_{k^{*}}
$$

plays the role of a complex diffusion coefficient ${ }^{1}$. If there are several saddle points, the one with the largest $v^{*}$ is the relevant one [6]. Since the growth rate $\operatorname{Im}\left(-i \omega(k)+i v^{*} k\right)$ in the comoving frame is maximal at $k^{*}$ for a relevant saddle point, the sign of $\operatorname{Re} \mathcal{D}$ is fixed:

$$
\operatorname{Re} \mathcal{D}>0 \text {. }
$$

Our analysis applies to sufficiently steep initial conditions [6]

$$
\lim _{x \rightarrow \infty} \phi(x, 0) e^{\lambda x}=0 \quad \text { for some } \lambda>\lambda^{*} ;
$$

initial conditions with bounded support fall into this class. An important result is that in a frame $\xi=x-v^{*} t$ moving with velocity $v^{*}$ to the right, the

$\overline{1} \mathcal{D}$ is the complex generalization of the diffusion constant $D$ as in [7] and should not be confused with the operator defined in Eq. (5.27) of [6]. 
asymptotic evolution of the field under the linear equation is given by

$$
\phi(x, t) \sim e^{-\lambda^{*} \xi+i q^{*} \xi-i \Omega\left(k^{*}\right) t} \frac{e^{-\xi^{2} / 4 \mathcal{D} t}}{\sqrt{4 \pi \mathcal{D} t}}
$$

where

$$
\Omega(k) \equiv \omega(k)-v^{*} k,
$$

and where the co-moving coordinate

$$
\xi=x-v^{*} t
$$

is held fixed while $t \rightarrow \infty$. This follows from the saddle point analysis of the Green's function in the limit of large $t$, cf. sections 5.3 and 5.5.1 in [6]. The saddle point equations (1) can be expressed in terms of $\Omega(k)$ as $\left.\mathrm{d}_{k} \Omega\right|_{k^{*}}=0$ and $\operatorname{Im} \Omega\left(k^{*}\right)=0$. For the remaining real part of $\Omega\left(k^{*}\right)$, we use the notation

$$
\Omega^{*}=\Omega\left(k^{*}\right)=\operatorname{Re} \Omega\left(k^{*}\right) .
$$

Eq. (5) illustrates that an initially sufficiently localized linear perturbation reaches the velocity $v^{*}$ and the spatial decay rate $\lambda^{*}$ for $t \rightarrow \infty$ under the dynamics of the linearized equation.

\subsection{Upper bounds on the velocity: proof of pulling}

When a front evolves under the full nonlinear equation into an unstable state, its asymptotic speed can never be smaller than the linear spreading velocity $v^{*}$. If the asymptotic speed equals $v^{*}$, the front is called pulled $[5,6,7,8]$, otherwise it is called pushed. As a rule of thumb, dynamical equations whose nonlinear terms are all suppressing the growth lead to pulled fronts, but there is at present no general theory that allows one to predict when fronts are pulled and when they are pushed.

In the present section, a simple proof is given that fronts in some pattern forming equations are pulled.

\subsubsection{A real field $\phi$ with nonlinearity $\mathcal{N}(\phi) \phi$}

Consider first an equation of motion for a real field $\phi$ 


$$
\partial_{t} \phi=\sum_{n=0}^{N} a_{n} \partial_{x}^{n} \phi-\mathcal{N}(\phi) \phi, \quad \mathcal{N}(0)=0
$$

with explicit linear terms and a nonlinearity ${ }^{2} \mathcal{N}(\phi) \phi$. Examples of such equations are the nonlinear diffusion equation $\partial_{t} u=\partial_{x}^{2} u+f(u)$, the SwiftHohenberg-equation

$$
\partial_{t} u=\varepsilon u-\left(\partial_{x}^{2}+1\right)^{2} u-u^{3}=(\varepsilon-1) u-2 \partial_{x}^{2} u-\partial_{x}^{4} u-u^{3}, \quad(\varepsilon>0),
$$

or the Extended Fisher-Kolmogorov (EFK) equation [7,23,24]

$$
\partial_{t} u=\partial_{x}^{2} u-\gamma \partial_{x}^{4} u+u-u^{3}
$$

The linear operator determines the dispersion relation $\omega(k)$ and the parameters $v^{*}, \Omega^{*}, q^{*}, \lambda^{*}$ and $\mathcal{D}$ as discussed above.

The relevant dynamics of a pulled front that leaves a homogeneous state behind $\left(\Omega^{*}=0=q^{*}\right)$, was identified in [6] by the leading edge transformation $\phi(x, t)=e^{-\lambda^{*} \xi} \psi(\xi, t), \xi=x-v^{*} t$. For pattern forming fronts with $\Omega^{*} \neq 0 \neq q^{*}$, different generalizations of this transformation are possible. While in the next section dealing with the asymptotic dynamics, the complete complex phase $e^{-\lambda^{*} \xi+i q^{*} \xi-i \Omega^{*} t}$ will be factored out of $\phi$, for deriving bounds, it will be more convenient here to factor out the envelope $e^{-\lambda^{*} \xi}$. In a frame moving with velocity $v^{*}$, the field $\hat{\psi}(\xi, t)$ is then defined through

$$
\phi(x, t)=e^{-\lambda^{*} \xi} \hat{\psi}(\xi, t), \quad \xi=x-v^{*} t .
$$

The effect of the transformation is demonstrated by comparing Fig. 1 with Fig. 2 below which show the original dynamical field $u$ of the Swift-Hohenberg equation and the associated field $\hat{\psi}$. The field $\hat{\psi}$ in Fig. 2 magnifies the relevant dynamics in the leading edge which we will analyze in section 3 , while this dynamics is hidden in Fig. 1.

With this transformation, the equation of motion for $\hat{\psi}$ becomes

$$
\partial_{t} \hat{\psi}-v^{*}\left(\partial_{\xi}-\lambda^{*}\right) \hat{\psi}=\sum_{n=0}^{N} a_{n}\left(\partial_{\xi}-\lambda^{*}\right)^{n} \hat{\psi}-\mathcal{N}\left(\hat{\psi} e^{-\lambda^{*} \xi}\right) \hat{\psi} .
$$

With the two auxiliary functions of the Fourier variable $k$

$\overline{2}$ Note that in [6] the complete nonlinear expression $\mathcal{N}(\phi) \phi$ was denoted as $N(\phi)$, but the present notation turns out to be more convenient for the generalizations. 


$$
\begin{aligned}
\sigma(k) & =\sum_{n=0}^{N} a_{n}\left(i k-\lambda^{*}\right)^{n}+v^{*}\left(i k-\lambda^{*}\right)=-i \omega\left(k+i \lambda^{*}\right)+i v^{*}\left(k+i \lambda^{*}\right), \\
\bar{\psi}(k, t) & =\int_{-\infty}^{\infty} \mathrm{d} \xi \hat{\psi}(\xi, t) e^{-i k \xi}
\end{aligned}
$$

the linear operators in Eq. (13) can be written in a more compact form

$$
\partial_{t} \hat{\psi}(\xi, t)=\int_{-\infty}^{\infty} \frac{\mathrm{d} k}{2 \pi} e^{i k \xi} \sigma(k) \bar{\psi}(k, t)-\mathcal{N}\left(\hat{\psi} e^{-\lambda^{*} \xi}\right) \hat{\psi}
$$

Now multiply the equation with $\hat{\psi}(\xi, t)$ and integrate over space. Using the identity

$$
\int \mathrm{d} k \sigma(k) \bar{\psi}(k, t) \bar{\psi}(-k, t)=\int \mathrm{d} k \operatorname{Re} \sigma(k)|\bar{\psi}(k, t)|^{2},
$$

the final result is

$$
\begin{aligned}
\frac{\partial}{\partial t} \int \mathrm{d} \xi \frac{\hat{\psi}^{2}(\xi, t)}{2}= & \int \frac{\mathrm{d} k}{2 \pi} \operatorname{Re} \sigma(k)|\bar{\psi}(k, t)|^{2} \\
& -\int \mathrm{d} \xi \mathcal{N}\left(\hat{\psi}(\xi, t) e^{-\lambda^{*} \xi}\right) \hat{\psi}^{2}(\xi, t)
\end{aligned}
$$

If $\phi$ initially is sufficiently steep (4) for $x \rightarrow \infty$, and if $|\phi|$ stays bounded behind the front at $x \rightarrow-\infty$, then the integrals exist initially. If furthermore the right hand side of (17) can be shown to be negative and of order $\int d \xi \hat{\psi}^{2}$, then $\int \mathrm{d} \xi \hat{\psi}^{2}(\xi, t) \downarrow 0$ for growing $t$. This means that in a frame moving with velocity $v^{*}, \hat{\psi}^{2}$ vanishes; and this implies that the front cannot move faster than $v^{*}$ for $t \rightarrow \infty$.

For the r.h.s. of (17) to be negative, we need both integrals to be negative. Since $\operatorname{Re} \sigma(k)=\operatorname{Im} \omega\left(k+i \lambda^{*}\right)-v^{*} \lambda^{*}$, the saddle point construction entails that $\operatorname{Re} \sigma\left(q^{*}\right)=0,\left.\partial_{k} \sigma\right|_{q^{*}}=0$ and $\left.\partial_{k}^{2} \sigma\right|_{q^{*}}=-2 \mathcal{D}$ with Re $\mathcal{D}>0$. Therefore

$$
\operatorname{Re} \sigma(k) \leq 0 \text { for all real } k \text {. }
$$

If there are several saddle points, this condition holds for the one corresponding to the largest spreading speed $v^{*}[6]$. The present formulation in terms of $\sigma(k)$ yields another route to this conclusion.

The sign of the integral over the nonlinearity is fixed if the sign of $\mathcal{N}$ is fixed. Therefore a sufficient criterion for the front to be pulled is

$$
\mathcal{N}(\phi) \geq 0 \text { for all relevant } \phi \text {. }
$$


In a pattern forming front, the sign of $\phi$ can change. This increases the relevant values of $\phi$ and therefore decreases the admissible functions $\mathcal{N}$. E.g., for $\mathcal{N}(\phi)=\phi^{r}$, a monotonic front with non-negative $\phi$ will be certainly pulled for all $r>0$, while for a pattern forming front, $r$ needs to be an even integer. Both in the Swift-Hohenberg and EFK equation, $\mathcal{N}$ is quadratic in the dynamical variable, hence the above argument immediately shows that sufficiently steep initial conditions lead to pulled fronts in these equations. With a few slight modifications, the analysis can also be extended to the difference equation $d C_{i} / d t=C_{i}-C_{i-1}^{2}$, for which fronts were empirically found to be pulled $[14,25]$.

\subsubsection{A complex field A: the complex Ginzburg-Landau-equation}

It was already remarked by Collet and Eckmann in a footnote in [22] that the above line of analysis can be extended to the case of the cubic Complex Ginzburg Landau equation. We present the argument here in our language, and then generalize it to an even more general class of equations in the next subsection.

We analyze the complex Ginzburg-Landau-equation for complex field $A(x, t)$

$$
\partial_{t} A=\epsilon A+\left(1+c_{1}\right) \partial_{x}^{2} A-\left(1-i c_{3}\right)|A|^{2} A, \quad \text { with } \epsilon, c_{1}, c_{3} \text { real, }
$$

or more generally an equation of the form

$$
\partial_{t} A=\sum_{n=0}^{N} a_{n} \partial_{x}^{n} A-\mathcal{N}(A) A, \quad \text { with } A(x, t), a_{n} \text { complex. }
$$

The saddle point parameters $\lambda^{*}, q^{*}, v^{*}, \Omega^{*}$ and $\mathcal{D}$ are again used for the transformation

$$
\hat{\psi}(\xi, t)=e^{-\lambda^{*} \xi} A(x, t), \quad \text { where } \xi=x-v^{*} t .
$$

The calculation now follows essentially the lines of the previous calculation except that one has to take into account that the field $\hat{\psi}$ and the coefficients are now complex. Therefore the equations of motion for $A^{*}$ or $\hat{\psi}^{*}$ have to be considered, too. They are, of course, derived by simply taking the complex conjugate of the equations for $A$ and $\hat{\psi}$. One then easily derives an equation for $\hat{\psi}^{*} \partial_{t} \hat{\psi}++\hat{\psi} \partial_{t} \hat{\psi}^{*}=\partial_{t}|\hat{\psi}|^{2}$ that after spatial integration and a few steps of calculation can be reduced to

$$
\frac{\partial}{\partial t} \int \mathrm{d} \xi \frac{|\hat{\psi}(\xi, t)|^{2}}{2}=\int \frac{\mathrm{d} k}{2 \pi} \operatorname{Re} \sigma(k)|\bar{\psi}(k, t)|^{2}-\int d \xi \operatorname{Re} \mathcal{N}(A)|\hat{\psi}|^{2} .
$$


Here $\bar{\psi}(k, t)$ and $\sigma(k)$ are defined precisely as in (14).

This means that the complex equation has been reduced to expressions that contain absolute values and real parts only. Therefore the conclusion from the previous subsection is easily extended: an equation of form (20) or (21) creates pulled fronts if

$$
\operatorname{Re} \mathcal{N}(A) \geq 0 \text { for all relevant } A \text {. }
$$

This is a nontrivial result, since in contrast to the real equation (12), the complex equation does not have an energy minimizing structure; still the bound can be derived in the same way as before. Specialized to the cubic Complex Ginzburg-Landau equation, the above analysis simply proves that fronts in this equation are pulled, a fact known already empirically since over 20 years $[7,26]$.

\subsubsection{Generalization of admissible linearities and nonlinearities}

In the last step, the admissible linear and nonlinear operators are reconsidered and generalized. For complex functions $A$, the general form is

$$
\mathcal{L} A+\mathcal{N}\left(A, \partial_{x} A, \partial_{x}^{2} A, \ldots, \partial_{x}^{m} A\right) A=0
$$

where $\mathcal{N}$ again can be complex. $\mathcal{L}$ is an arbitrary complex linear operator that can take the differential form above, but also a difference or integral or mixed form as discussed in Section V of [6]. It determines the saddle point parameters $v^{*}, \lambda^{*}, q^{*}$ and $\mathcal{D}$. Independent of the original functional form of the linear operator, the expansion about the (large- $t$, large- $x$ )-saddle point will lead to the differential form

$$
\tau_{0} \partial_{t} \hat{\psi}=\ldots-\mathcal{N}\left(A, \partial_{x} A, \partial_{x}^{2} A, \ldots, \partial_{x}^{m} A\right) \hat{\psi}
$$

The analysis now proceeds as before with the final result

$$
\frac{\partial}{\partial t} \int \mathrm{d} \xi \frac{|\hat{\psi}(\xi, t)|^{2}}{2}=\ldots-\int \mathrm{d} \xi \operatorname{Re} \frac{\mathcal{N}\left(A, \partial_{x} A, \ldots, \partial_{x}^{m} A\right)}{\tau_{0}}|\hat{\psi}|^{2}
$$

A sufficient criterion for the front to be pulled is

$$
\operatorname{Re} \frac{\mathcal{N}\left(A, \partial_{x} A, \ldots, \partial_{x}^{m} A\right)}{\tau_{0}} \geq 0 \text { for all relevant } A
$$


In essence, the method discussed here confirms mathematically what one would expect intuitively for equations where only the linear terms lead to growth away from the unstable state $\phi=0$, while all the nonlinear terms are clearly stabilizing. In such cases, fronts are shown to be of the pulled type. There are several cases where fronts are empirically known to be pulled, but where the method in its present formulation fails. E.g., while adding a nonlinearity like $-\left(\partial_{x} u\right)^{2} u$ to the Swift-Hohenberg equation (10) or EFK equation (11) leaves the fronts in these equations of the pulled type, since $\mathcal{N}=\left(\partial_{x} u\right)^{2} \geq 0$, the nonlinearity of the Kuramoto-Sivashinsky-equation $\partial_{t} u=-\partial_{x}^{2} u-\partial_{x}^{4} u+\left(\partial_{x} u\right) u$ does not fall into the class (28). In fact, extending the method to the Kuramoto-Sivashinsky equation must clearly be quite a challenge, since adding a linear term $c \partial_{x}^{3} u$ gives a transition to pushed fronts for $c \approx 0.15[7]$. An easier challenge to start with appears to be the the Cahn-Hilliard-equation $\partial_{t} u=-\partial_{x}^{2}\left(\partial_{x}^{2} u+u-u^{3}\right)$. Again, in its present form our method does not apply straightforwardly to the Cahn-Hilliard equation. Nevertheless, for a front penetrating the state $u=0$ under the Cahn-Hilliard dynamics, we derive after a few partial integrations that

$$
\partial_{t} \int \mathrm{d} \xi \hat{\psi}^{2}=\ldots-3 \int \mathrm{d} \xi \hat{\psi}^{2}\left(\left(\partial_{x} u\right)^{2}-\left(\lambda^{*} u\right)^{2}\right) .
$$

It is very likely that the sign of this integral over the nonlinearity is negative, since $\left(\partial_{x} u\right) / u$ is the local slope of the full oscillating front, while $\left(\lambda^{*} u\right) / u$ is the slope of only the envelope in the leading edge. However, we have not yet been able to prove this.

In summary, we have derived sufficient criteria for a large class of equations to form pulled fronts, i.e., fronts that propagate with the linear spreading speed $v^{*}$. We now proceed to determining their actual rate of convergence to the asymptotic behavior.

\section{Power law convergence to the asymptotic speed and shape of a pulled front}

In [6] we have analyzed pulled fronts that for long times approach uniformly translating fronts, and we have derived their rate of convergence to the asymptotic velocity and front profile. We will now extend this analysis to pattern forming fronts.

Our analysis in [6] was based on a complete matching of the transient dynamics in the leading edge (where the nonlinearities in the dynamical equation can be neglected) to the behavior in the nonlinear front region itself. This detailed analysis explicitly demonstrates that the matching procedure can be carried 
out order by order. It is remarkable and in line with the picture that has emerged for the pulled front mechanism, that the coefficients in the asymptotic expressions are actually obtained from the asymptotic analysis in the leading edge only; more precisely they are given by the saddle point parameters (1), (2) of the linearized equation. This is because for the analysis in the leading edge only input on the dominant analytic behavior of the asymptotic front profile is needed ${ }^{3}$. For brevity, we will therefore present here only the generalization of the asymptotic expansion in the leading edge, following the lines of our earlier paper.

\subsection{The dynamical equation for the leading edge variable $\psi$ in the frame $\xi_{X}$}

The first ingredient of the asymptotic analysis for the front convergence is to note that in the leading edge, the saddle point analysis from Section 2.1 implies that the field $\psi(\xi, t)$ defined through

$$
\phi(x, t)=e^{-\lambda^{*} \xi} e^{i q^{*} \xi-i \Omega^{*} t} \psi(\xi, t), \quad \xi=x-v^{*} t .
$$

becomes a function which varies slowly in space and time for large $x$ and $t$, and this slow dynamics is governed by a generalized diffusion equation of the form

$$
\frac{\partial \psi}{\partial t}=\mathcal{D} \frac{\partial^{2} \psi}{\partial \xi^{2}}+\mathcal{D}_{3} \frac{\partial^{3} \psi}{\partial \xi^{3}}+w \frac{\partial^{2} \psi}{\partial t \partial \xi}+\tau_{2} \frac{\partial^{2} \psi}{\partial t^{2}}+\cdots-\mathcal{N}(\phi, \ldots) \psi .
$$

In the function $\psi$, the full complex prefactor is factorized out of $\phi$, in contrast to the partial factorization in Eq. (13). The parameter $\mathcal{D}$ is the generalized diffusion coefficient defined already in Eq. (2) above. Likewise the other expansion coefficients $\mathcal{D}_{3}, w, \tau_{2}$ et cetera can all be expressed in terms of the expansion of the dispersion relation near the saddle point - see Eq. (5.64) of [6]. E.g., we simply have $\mathcal{D}_{3}=(1 / 3$ ! $) \mathrm{d}^{3} \omega /\left.\mathrm{d} k^{3}\right|_{k^{*}}$. Note that we call Eq. (31) a generalized diffusion equation since the dominant terms for large $\xi$ and $t$ are in fact diffusive and can generate the Gaussian from Eq. (5).

For equations which lead to uniformly translating fronts, $q^{*}=0$ and $\mathcal{D}$ is real, but for pattern forming fronts $\mathcal{D}$ is generally complex and $q^{*} \neq 0$.

As discussed in $[5,6,7]$, if we follow a level line where $|\phi|$ is constant, the $1 / \sqrt{t}$ term in (5) implies an unbounded logarithmic shift in the position of the level line, and hence of the transient fronts in the nonlinear equation. The crux of

3 In the language of a matching analysis, the outer (leading edge) expansion of the inner (nonlinear front) solution is expressed by the condition (42) below. 
the convergence analysis is therefore to introduce a collective coordinate $X(t)$ for the front position,

$$
\dot{X}(t)=\frac{c_{1}}{t}+\frac{c_{3 / 2}}{t^{3 / 2}}+\frac{c_{2}}{t^{2}}+\cdots \quad \Longleftrightarrow X(t)=c_{1} \ln t-\frac{2 c_{3 / 2}}{t^{1 / 2}}+\cdots,
$$

and to perform an expansion in the logarithmically shifted frame

$$
\xi_{X}=\xi-X(t)=x-v^{*} t-X(t) .
$$

For pattern forming fronts, we likewise introduce a global time-dependent phase $\Gamma(t)$,

$$
\dot{\Gamma}(t)=\frac{d_{1}}{t}+\frac{d_{3 / 2}}{t^{3 / 2}}+\frac{d_{2}}{t^{2}}+\cdots \quad \Longleftrightarrow \Gamma(t)=d_{1} \ln t-\frac{2 d_{3 / 2}}{t^{1 / 2}}+\cdots,
$$

and we define the field $\psi_{X}$ in the shifted frame $\xi_{X}$ and with a global slow phase factor $\Gamma$ by writing $\phi$ as

$$
\phi(x, t)=e^{-\lambda^{*} \xi_{x}} e^{i q^{*} \xi_{X}-i\left(\Omega^{*} t+\Gamma(t)\right)} \psi_{X}\left(\xi_{X}, t\right) .
$$

Comparison of (30) and (35) shows that

$$
\psi(\xi, t)=e^{\lambda^{*} X(t)-i q^{*} X(t)-i \Gamma(t)} \psi_{X}\left(\xi_{X}, t\right) .
$$

With this transformation, we obtain from (31) the relevant dynamical equation $^{4}$ for $\psi_{X}\left(\xi_{X}, t\right)$

$$
\begin{array}{r}
\frac{\partial \psi_{X}}{\partial t}-\dot{X}(t)\left(i k^{*}+\frac{\partial}{\partial \xi_{X}}\right) \psi_{X}-i \dot{\Gamma}(t) \psi_{X}=\mathcal{D} \frac{\partial^{2} \psi_{X}}{\partial \xi_{X}^{2}}+\mathcal{D}_{3} \frac{\partial^{3} \psi_{X}}{\partial \xi_{X}^{3}}+\ldots \\
+w\left[\frac{\partial}{\partial t}-\dot{X}(t)\left(i k^{*}+\frac{\partial}{\partial \xi_{X}}\right)-i \dot{\Gamma}(t)\right] \frac{\partial \psi_{X}}{\partial \xi_{X}}+\cdots-\mathcal{N} \psi_{X} .
\end{array}
$$

\footnotetext{
4 The term proportional to $w$ is not present for equations like the Swift-Hohenberg equation or for the Complex Ginzburg Landau equation, but can be present in more general cases. As was already found for uniformly translating fronts [6], this term does not affect the relevant terms for the power law relaxation.
} 
3.2 The asymptotic expansion for $\psi_{X}$ in terms of similarity variables of the diffusion equation

As we already pointed out above, in dominant order, the dynamical equation (31) for $\psi(\xi, t)$ is a diffusion equation, and this was reflected by the fact that in the fully linear spreading problem, $\psi(\xi, t)$ is just the fundamental Gaussian similarity solution $e^{-\xi^{2} /(4 \mathcal{D} t)} / \sqrt{t}-$ Cf. Eq. (5). As explained in $[6,16]$, the nonlinearity in (31) can be interpreted as a sink for the diffusive field $\psi_{X}$ to the left of the leading edge. This imposes that in contrast to the linear problem, $\psi$ has to increase linearly in $\xi$ for small $\xi$. The relevant fundamental solution of the diffusion equation which has this behavior is

$$
\psi(\xi, t) \sim \frac{\xi}{t^{3 / 2}} e^{-\xi^{2} /(4 \mathcal{D} t)},
$$

and as explained in detail in $[6,7]$ one can already obtain the dominant term of the power law relaxation of the velocity and front shape from this argument.

The expansion is systematized by working in the $\xi_{X}$ frame, as explained above, and by recognizing that the similarity variable of the diffusion equation is

$$
z=\frac{\xi_{X}^{2}}{4 \mathcal{D} t}
$$

In short, since far ahead of the front in the leading edge, $\psi_{X}$ will fall off like a Gaussian $e^{-z}=e^{-\xi^{2} /(4 \mathcal{D} t)}$ for a sufficiently steep front (4) (see [6]), we write

$$
\psi\left(\xi_{X}, t\right)=G(z, t) e^{-z}
$$

To ensure the Gaussian decay for large $\xi_{X}$ and finite $t$, we require

$$
\lim _{z \rightarrow \infty} G(z, t) e^{-z}=0 \quad \Longleftrightarrow \quad \lim _{\xi_{x} \rightarrow \infty} \psi_{X}\left(\xi_{X}, t\right)=0
$$

Note that as we already stated in $(3), \operatorname{Re} \mathcal{D}>0$, so the limit $z \rightarrow \infty$ should be taken along a line in the right complex $z$ plane. This is the first boundary condition for $G$. The second boundary or matching condition arises from the behavior for small $\xi_{X}$, actually in the transition towards the nonlinear regime. In agreement with the intuitive argument about the nonlinearity as a sink for the diffusion process, one derives

$$
\psi_{X}\left(\xi_{X}, t\right) \stackrel{\xi_{X} / \sqrt{t} \rightarrow 0}{=} \alpha \xi+\beta \quad \Longleftrightarrow \quad G(z, t)=2 \alpha \sqrt{\mathcal{D} z t}+\ldots
$$


where $\alpha$ and $\beta$ are in general complex constants with $\alpha \neq 0$ due to the nonlinearity ${ }^{5}$.

Upon substitution of (40) into Eq. (37) for $\psi_{X}$, and using the expansion (32) for $X(t)$ and (34) for $\Gamma(t)$, we obtain the equation of motion for $G$

$$
\begin{aligned}
t \partial_{t} G- & \left(c_{1}+\frac{c_{3 / 2}}{\sqrt{t}}\right)\left[i k^{*}+\frac{\sqrt{z}}{\sqrt{\mathrm{D} t}}\left(\partial_{z}-1\right)\right] G-i\left(d_{1}+\frac{d_{3 / 2}}{\sqrt{t}}\right) G= \\
{\left[z \partial_{z}^{2}+\left(\frac{1}{2}-z\right) \partial_{z}-\frac{1}{2}\right] G } & \\
& +\frac{\mathcal{D}_{3} \sqrt{z}}{\mathcal{D}^{\frac{3}{2}} \sqrt{t}}\left[\frac{3}{2}\left(\partial_{z}-1\right)^{2}+z\left(\partial_{z}-1\right)^{3}\right] G \\
& +w \frac{\sqrt{z}}{\sqrt{\mathcal{D} t}}\left[t \partial_{t}-z\left(\partial_{z}-1\right)-1-i k^{*} c_{1}-i d_{1}\right]\left(\partial_{z}-1\right) G+\cdots
\end{aligned}
$$

The relevant long-time asymptotics of $\psi_{X}$ then directly follows from solving this equation with boundary conditions (41) and (42) [6]. As in [6], the coefficients $c_{i}$ and $d_{i}$ in $X(t)$ and $\Gamma(t)$ can be obtained by expanding $G(z, t)$ as an asymptotic series in terms of functions of the similarity variable $z$,

$$
G(z, t)=t^{1 / 2} g_{-1 / 2}(z)+g_{0}(z)+\frac{g_{1 / 2}(z)}{t^{1 / 2}}+\frac{g_{1}(z)}{t} \cdots, \quad(t \gg 1),
$$

where the matching condition (42) implies that the leading order indeed is $\sqrt{t}$ with the coefficient $g_{-1 / 2}(z)=\sqrt{z}+\ldots$ for small $z$.

From here on, the analysis is just the technical implication of the expansion introduced above. Since the structure of the analysis follows essentially the one given in our earlier work on uniformly translating fronts, we relegate the details to appendix A. The final outcome of the analysis is that the velocity relaxes to $v^{*}$ according to the general formula

$$
v(t) \equiv v^{*}+\dot{X}(t)=v^{*}-\frac{3}{2 \lambda^{*} t}+\frac{3 \sqrt{\pi}}{2\left(\lambda^{*}\right)^{2} t^{3 / 2}} \operatorname{Re} \frac{1}{\sqrt{\mathcal{D}}}+\mathcal{O}\left(\frac{1}{t^{2}}\right)
$$

5 For the nonlinear diffusion equation, we derived $\mathcal{D} \alpha=\int_{-\infty}^{\infty} d \xi \mathcal{N} \psi$ in section 2.5.2 of [6]. The relation between non-vanishing $\alpha$ and $\mathcal{N}$ can be generalized to pattern forming fronts [16]. In general, $\mathcal{N}$ then becomes time dependent and some temporal averaging is required. For the cubic CGL equation (20), however, we obtain $\mathcal{D} \alpha=\int_{-\infty}^{\infty} \mathrm{d} \xi\left(1+i c_{3}\right)|A|^{2} \psi(\xi)$ without temporal averaging. The phase of $\alpha$ changes in the same way as the phase of $\psi$ while the complete problem is phase invariant. 
while the phase relaxation is governed by a similar expression,

$$
\dot{\Gamma}(t)=-q^{*} \dot{X}(t)-\frac{3 \sqrt{\pi}}{2 \lambda^{*} t^{3 / 2}} \operatorname{Im} \frac{1}{\sqrt{\mathcal{D}}}+\mathcal{O}\left(\frac{1}{t^{2}}\right)
$$

\subsection{Convergence of a coherent front profile to its asymptotic shape}

The above expressions are valid for any pulled front, irrespective of whether it is asymptotically uniformly translating or a coherent or incoherent pattern forming front ${ }^{6}$. Here 'coherent' means that the approximately periodic pattern laid down by the leading edge of the front stays periodic in the nonlinear region, while incoherent means that the pattern undergoes some further dynamics behind the front. Such incoherent fronts arise e.g. in some parameter regimes of the cubic and quintic Complex Ginzburg Landau equation $[7,16,26,27]$ or the Kuramoto-Sivashinsky equation [7]. Even when a pulled pattern forming front is incoherent the linear dynamics in the leading edge is described by the above equations. The dynamics in the leading edge is therefore still coherent: the incoherent behavior only sets in in the region where the dynamics become truly nonlinear. Since the matching condition which the nonlinear dynamics imposes on the linear leading edge dynamics is still the same in this case $[7,16]$, the above results even apply to incoherent fronts. However, the phase relaxation applies in that case only to the coherent dynamics in the leading edge.

If the pattern forming front is coherent, the results apply throughout the whole front region. More precisely, we call a front coherent if the asymptotic front solution is time periodic in the co-moving frame $\xi=x-v^{*} t$, i.e. if there is some period $T$ such that

$$
\Phi(\xi, t+T)=\Phi(\xi, t), \quad \text { where } \phi(\xi, t) \stackrel{t \rightarrow \infty}{=} \Phi(\xi, t) .
$$

The dynamics of the leading edge actually determines this period to be

$$
T=2 \pi / \Omega^{*},
$$

where $\Omega^{*}$ is the frequency determined by the saddle point (1). This can be easily read from Eq. (5) or from Eq. (35) and the knowledge that $\psi_{X}\left(\xi_{X}, t\right)$ becomes stationary for $t \rightarrow \infty$.

${ }^{6}$ Of course, for uniformly translating fronts there is no phase, hence $q^{*}=0=\Omega^{*}$ and $\operatorname{Im} \mathcal{D}=0$ in (46). 
Because of the temporal periodicity, we can generally write a coherent $\Phi$ in the whole spatial domain as a Fourier series

$$
\Phi(\xi, t)=\sum_{n=0, \pm 1, \ldots} e^{-i n \Omega^{*} t} \Phi^{n}(\xi)
$$

In our analysis [6] of fronts which converge to a uniformly translating front solution, we explicitly showed that to order $\mathcal{O}\left(1 / t^{2}\right)$, the front shape relaxation follows the velocity relaxation adiabatically. An extension of the analysis to coherent pattern forming fronts shows that a similar result holds for these. The reason is that when the front is converging to its asymptotic shape as $1 / t$, the temporal derivative terms in the dynamical equations only generate terms of order $1 / t^{2}$ in the asymptotic expansion, while the terms coming from the adiabatic variation of $v(t)$ and $\Gamma(t)$ generate terms of order $1 / t$ and $1 / t^{3 / 2}$. In other words, to order $1 / t^{3 / 2}$ the only temporal dependence comes in parametrically via $v(t)$ and $\Gamma(t)$. Thus, for long times, coherent pattern forming fronts relax to their asymptotic shape according to

$$
\left.\phi(x, t) \stackrel{t \gg 1}{=} \Phi_{v(t)}\left(\xi_{X}, t\right)+\mathcal{O}\left(t^{-2}\right) \text { with } \Phi_{(} \xi_{X}, t\right) \approx \Phi_{v(t)}\left(\xi_{X}, t+T(t)\right),
$$

where $v(t)$ and $\Gamma(t)$ are given by Eqs (45) and (46) above, and where $T(t)$ is the instantaneous period $2 \pi /\left(\Omega^{*}+\dot{\Gamma}(t)\right)$. In terms of the temporal Fourier series this result can be written as

$$
\phi(x, t) \stackrel{t \gg 1}{=} \sum_{n=0, \pm 1, \cdots} e^{-i n\left(\Omega^{*} t+\Gamma(t)\right)} \Phi_{v(t)}^{n}\left(\xi_{X}\right)+\mathcal{O}\left(t^{-2}\right)
$$

where the $\Phi_{v}^{n}$ are the Fourier transform functions of the coherent pattern forming solutions ${ }^{7}$ with velocity $v$ and frequency $\Omega^{*}+\dot{\Gamma}$. Thus the above result expresses that the coherent front profiles follow this family of solutions adiabatically, and that their velocity and frequency shift $\dot{\Gamma}$ is set completely by the dynamics in the leading edge.

\footnotetext{
7 Clearly, this result implies the existence of a two-parameter family of coherent front solutions, parametrized by their velocity and frequency. It is argued in [7] that this is the generic case, and that if such a two-parameter family of solutions does not exist, there generically does not exist a coherent pulled front solution either; the fronts will then be incoherent.
} 


\section{Numerical study of the relaxation behavior of fronts in the Swift- Hohenberg equation}

We now illustrate the above analysis with numerical results obtained for the Swift-Hohenberg equation (10). This equation has often been used $[17,18,19,20,21,22]$ as one of the simplest equations to illustrate the behavior of coherent pattern forming fronts. Collet and Eckmann were the first to prove that fronts propagating into the linearly unstable state $\phi=0$ are pulled; the analysis of section 2 applies too and therefore establishes this fact as well. In the simulations of this equation presented here, we study the approach of the fronts to these asymptotic pulled front solutions, starting from a Gaussian initial condition. Note in this regard that while the Swift-Hohenberg is often studied for small $\varepsilon$ where the dynamics maps onto an amplitude expansion, our front convergence analysis applies generally. We will illustrate this by taking finite values of $\varepsilon$. Fig. 1 shows a $\phi$-profile for $\varepsilon=0.5$.

We first illustrate an important ingredient of our convergence analysis. As we argued above, in the co-moving frame $\xi=x-v^{*} t$ the leading edge variable $\psi$ defined in (30) should asymptotically behave as $\xi /\left(t^{3 / 2}\right) e^{-\xi^{2} /(4 D t)}$ [Cf. Eq. (38)]. To illustrate this for the Swift-Hohenberg equation, we show in Fig. 2 three snapshots of the leading edge variable $t^{3 / 2} \hat{\psi}(x, t)=e^{\lambda^{*}\left(x-v^{*} t\right)} \phi(x, t)$ in a simulation for $\varepsilon=0.5$; according to our analysis, the envelope of this function should asymptotically behave as

$$
\left(x-v^{*} t\right) e^{-\left(x-v^{*} t\right)^{2} /(4 D t)}, \quad \text { with } \frac{1}{D} \equiv \operatorname{Re} \frac{1}{\mathcal{D}} .
$$

Our numerical results in Fig. 2 fully confirm this behavior.

To test our convergence results, we have to extract the velocity $v(t)$ and frequency $\Omega^{*}+\dot{\Gamma}(t)$ from our numerical data. Because of the oscillating character of the fronts, this is nontrivial in principle. We will do it in a pragmatic way, replacing differentials by finite difference approximants: In our simulation, we keep track of the local maxima of $\phi(x, t)$ and from these determine the positions $X_{n}$ and times $t_{n}$ at which the foremost maximum $n$ reaches a predetermined fixed "level" $\ell$. From this we calculate the finite difference approximants

$$
v_{\ell}\left(t_{n}\right)=\frac{X_{n}-X_{n-1}}{t_{n}-t_{n-1}}, \quad \Omega_{\ell}\left(t_{n}\right)=\frac{2 \pi}{t_{n}-t_{n-1}} .
$$

and then analyze whether indeed the convergence of these quantities to their asymptotic values is consistent with the universal $\ell$-independent behavior derived above. The error of the finite difference approximants is of $\mathcal{O}\left(1 / t^{2}\right)$ only. 


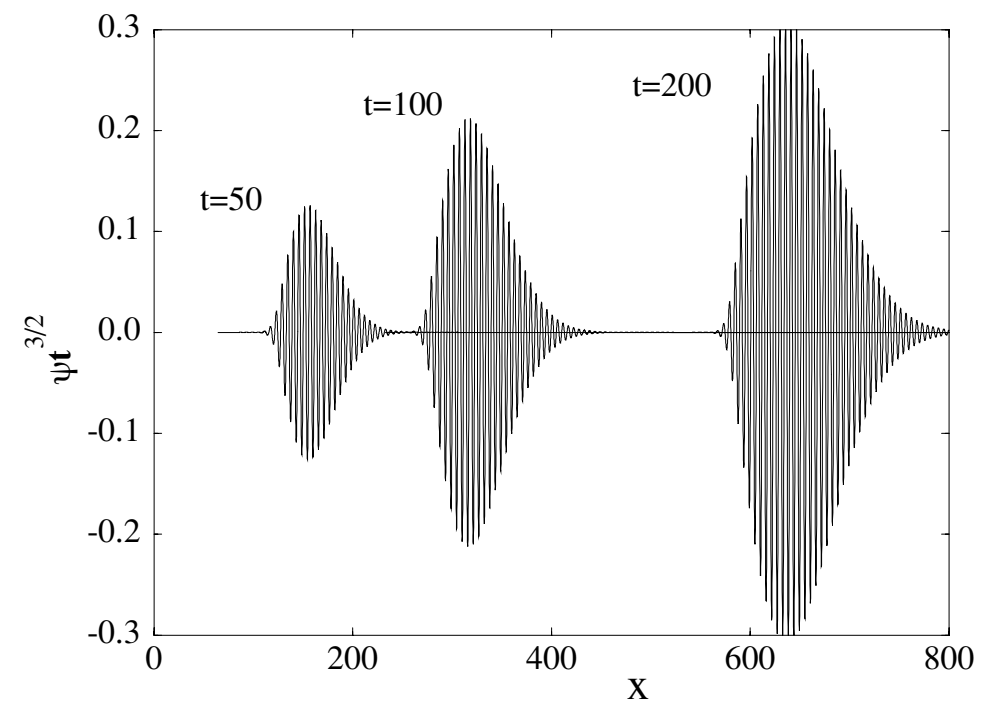

Fig. 2. Three snapshots of the function $t^{3 / 2} \hat{\psi}$ obtained from our simulations of the Swift-Hohenberg equation for $\varepsilon=0.5$. The results confirm the asymptotic behavior (52). Note in particular the diffusive broadening of the pattern: the one at time $t=200$ is twice as wide as the one at time $t=50$.

For testing the convergence up to terms of $\mathcal{O}\left(1 / t^{3 / 2}\right)$, the discretization error is therefore irrelevant.

In Fig. 3 we show two plots of the velocity relaxation data for two different values of $\varepsilon$, namely $\varepsilon=0.5$ and $\varepsilon=5$. The various lines indicate the velocity extracted for different levels $\ell$. To probe the predicted behavior in detail, we have plotted $v_{\ell}(t)-v^{*}-c_{1} / t$ versus $t^{-3 / 2}$. According to our prediction (45) this velocity difference should asymptotically approach 0 along the dashed lines. Similar plots for the frequency relaxation, obtained from the same runs, are shown in Fig. 4. Clearly, all our numerical results are in full agreement with the predicted behavior.

We finally study the convergence of the shape of the profile to its asymptotic form. In principle, the information is contained in the expression (51) above, but to make it explicit one would have to know all functions $\Phi_{v}^{n}$. Since our goal here is simply to check that the shape relaxation follows the velocity and phase relaxation adiabatically, we circumvent this problem as follows. We construct an effective (real) envelope $A\left(\xi_{X}, t\right)$ of the front profile ${ }^{8}$ in the comoving frame by tracking the positions of the maxima of $\phi(x, t)$ during one effective period $2 \pi /\left(\Omega^{*}+\dot{\Gamma}(t)\right)$. In doing so, $\xi_{X}$ is determined by requiring that $A\left(\xi_{X}=0, t\right)=$ const. where the constant is chosen so that the level of the effective envelope at this point is about half of its asymptotic value. The implication of (51) now is that the convergence of the effective envelope $A(\xi, t)$ determined this way should, up to terms of $\mathcal{O}\left(1 / t^{2}\right)$, adiabatically follow the

8 Note that this real envelope $A$ differs from the complex amplitude $A$ of the previous sections. 
(a)

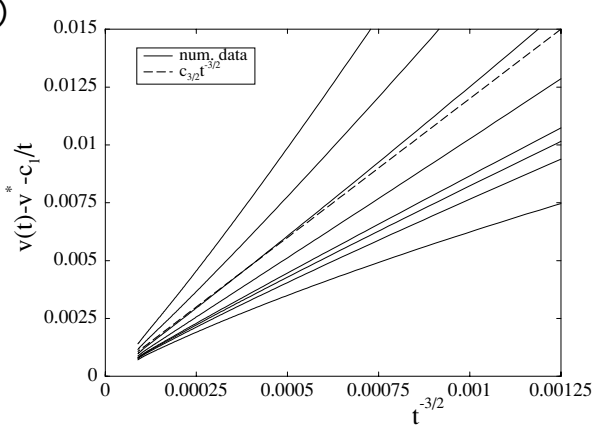

(b)

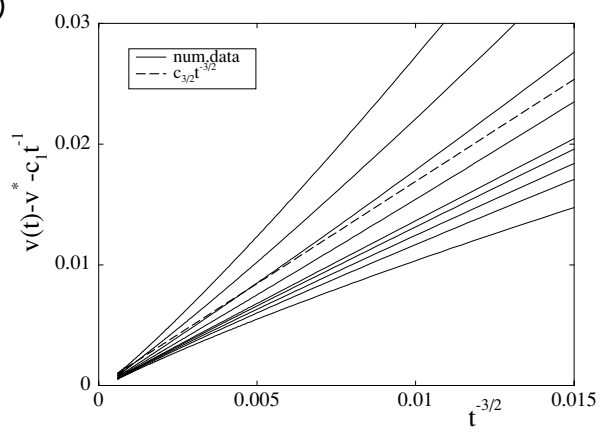

Fig. 3. Velocity difference $v_{\ell}(t)-v^{*}-c_{1} / t$ as a function of $t^{-3 / 2}$ for $\varepsilon=0.5$ (panel a) and $\varepsilon=5$ (panel b). The various lines denote, from top to bottom, the levels $\ell=0.0001 \sqrt{\varepsilon}, 0.001 \sqrt{\varepsilon}, 0.01 \sqrt{\varepsilon}, 0.05 \sqrt{\varepsilon}, 0.2 \sqrt{\varepsilon}, 0.3 \sqrt{\varepsilon}$ and $0.5 \sqrt{\varepsilon}$. The dashed line is the asymptotic slope according to the exact expression (45).

(a)

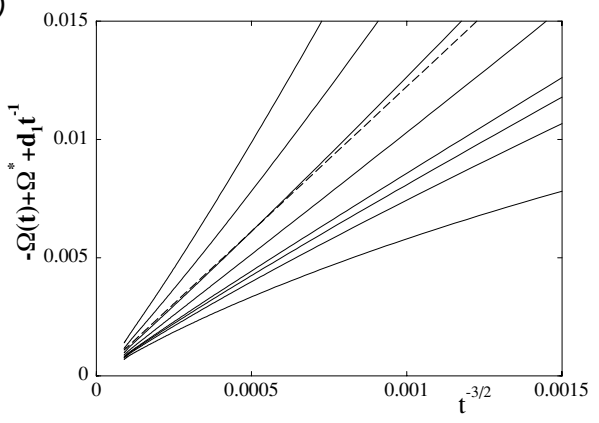

(b)

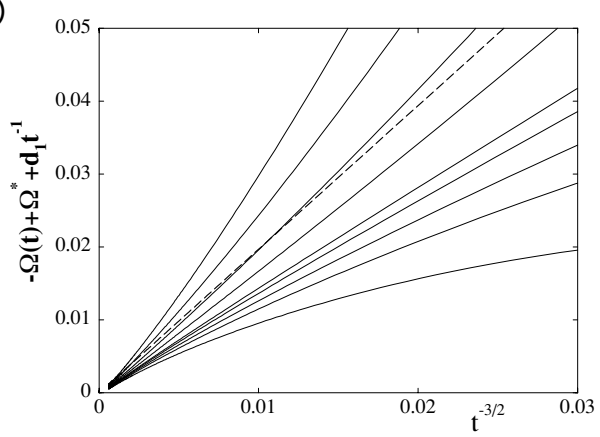

Fig. 4. As Fig. 3, but now for the frequency relaxation $\Omega(t)=\Omega^{*}+\dot{\Gamma}(t)$. velocity and shape relaxation:

$$
A\left(\xi_{X}, t\right)=A_{v(t), \dot{\Gamma}(t)}\left(\xi_{X}\right)+\mathcal{O}\left(1 / t^{2}\right),
$$

so that

$$
\begin{aligned}
A\left(\xi_{X}, t\right)-A\left(\xi_{X}, t^{\prime}\right)= & \frac{\delta A_{v, \dot{\Gamma}}\left(\xi_{X}\right)}{\delta v}\left[v(t)-v\left(t^{\prime}\right)\right] \\
& +\frac{\delta A_{v, \dot{\Gamma}}\left(\xi_{X}\right)}{\delta \dot{\Gamma}}\left[\dot{\Gamma}(t)-\dot{\Gamma}\left(t^{\prime}\right)\right]+\mathcal{O}\left(1 / t^{2}\right) .
\end{aligned}
$$

As in the discretization (53), the averaging over one period only affects the terms of $\mathcal{O}\left(1 / t^{2}\right)$ in this expression.

Fig. 5 shows the effective envelope $A\left(\xi_{X}, t\right)$ for the front from Fig. 1. The figure confirms that even for this value, where the pattern behind the front is rapidly oscillating, the effective envelope can be obtained accurately and is smooth.

In Fig. 6 we present our analysis of the large-time shape relaxation of this 


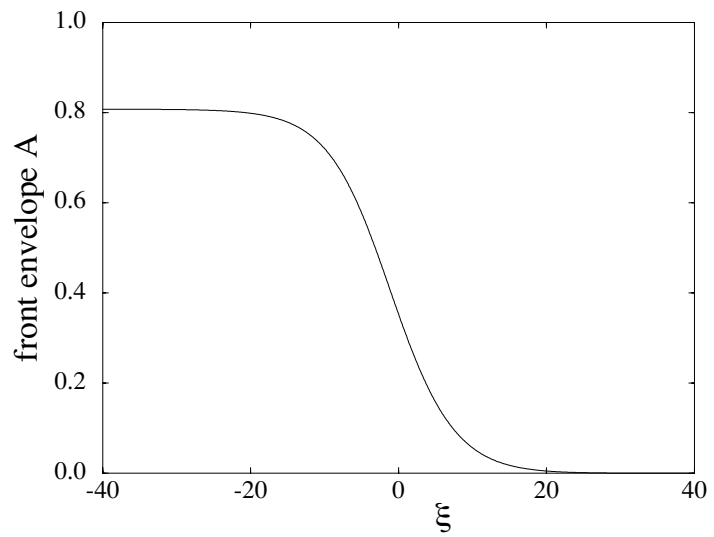

Fig. 5. The front envelope $A\left(\xi_{X}, t\right)$ for $\varepsilon=0.5$ obtained as described in the text. In this case, $t=160$, and the front shape is obtained by averaging over one period that lasts about $\Delta t=2$. Note the different horizontal scale in comparison with Fig. 1 .

(a)

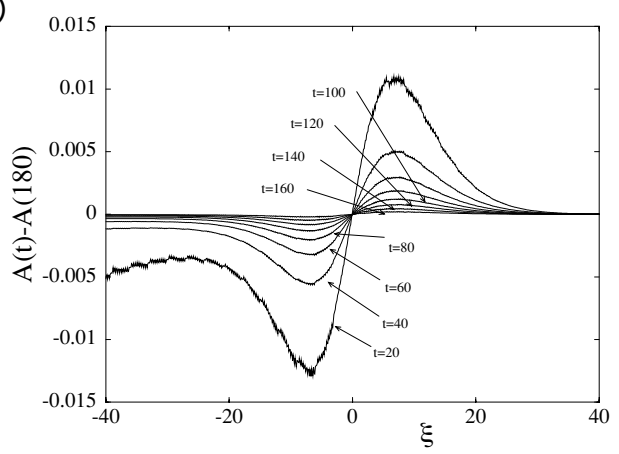

(b)

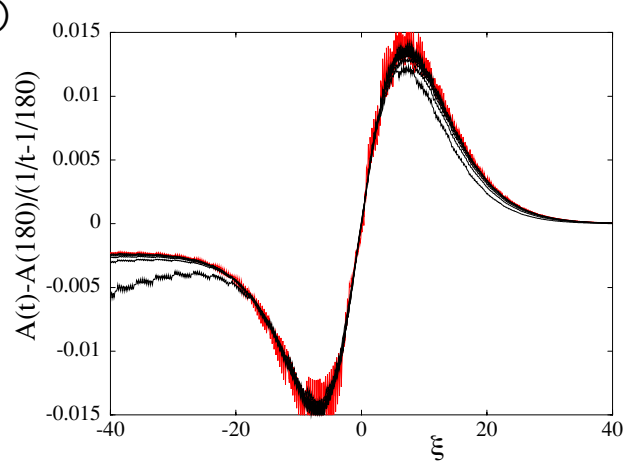

Fig. 6. (a) The convergence of the effective envelope difference $A\left(\xi_{X}, t\right)-A\left(\xi_{X}, 180\right)$, as obtained from the numerical solutions illustrated in Fig. 5. (b) The ratio (56) as obtained from the data shown in panel (a). The figure confirms that this ratio converges to a time-independent function, in agreement with our predictions.

profile. Panel (a) shows the difference $A\left(\xi_{X}, t\right)-A\left(\xi_{X}, 180\right)$, while in panel (b) we plot the ratio

$$
\frac{A\left(\xi_{X}, t\right)-A\left(\xi_{X}, 180\right)}{1 / t-1 / 180},
$$

which according to our prediction (55) should for large times become a function of $\xi_{X}$ only. It is clear that our numerical results fully corroborate this. 


\section{Conclusion}

In this paper we have presented two types of results. First of all, we have introduced a simple line of analysis which allows us to prove for certain classes of equations which include the Swift-Hohenberg equation, the Extended FisherKolmogorov equation and the cubic Complex Ginzburg Landau equation that fronts are pulled. The method works for real or complex equations and fields and is not restricted to nonlinearities like $\mathcal{N}(A) A=|A|^{2 n} A$ with integer $n$, but also can treat nonlinearities that depend, e.g., on $\partial_{x} A$. Important is that the over-all sign of $\operatorname{Re} \mathcal{N}$ can be determined.

Second, we have derived the universal slow convergence of the velocity and phase of coherent pattern forming pulled fronts to their asymptotic value. Numerical simulations of the Swift-Hohenberg equation are in full agreement with these predictions. In another paper [16], we have shown that the results for the velocity convergence also apply to incoherent pattern forming fronts.

We are grateful to Kees Storm for many useful discussions. 


\section{A Derivation of Eqs. (45) and (46)}

The derivation follows essentially the lines of [6], except that $z$ is now a complex rather than a real variable, and that there are additional terms due to $q^{*}$ and $\dot{\Gamma}$. The task is to solve (43) with the ansatz (44) and with boundary conditions (41) and (42). Actually, the analysis of the nonlinear region for finite $t$ contributes additional terms to (42) which will play a role in the calculation of the subleading terms. The boundary conditions for $\psi_{X}$ become

$$
\begin{aligned}
& \psi_{X}\left(\xi_{X}, t\right)=\alpha \xi_{X}+\beta+\frac{f_{1}\left(\xi_{X}\right)}{t}+O\left(\frac{f_{3 / 2}\left(\xi_{X}\right)}{t^{3 / 2}}\right), \\
& \psi_{X}\left(\xi_{X}, t\right) \stackrel{\xi_{X}^{2} /(4 \mathcal{D} t) \gg 1}{\longrightarrow} 0 .
\end{aligned}
$$

Insertion into the ansatz (44) implies for the function $G(z, t)$ that

$$
\begin{aligned}
& G(z, t)=\sqrt{t}\left[2 \alpha \sqrt{\mathcal{D} z}+O\left(z^{3 / 2}\right)\right]+[\beta+O(z)]+\frac{O(\sqrt{z})}{\sqrt{t}}+O\left(\frac{1}{t}\right) \\
& \lim _{z \rightarrow \infty} e^{-z} G(z, t)=0 .
\end{aligned}
$$

These boundary conditions determine a unique solution for the functions $g_{1 / 2}(z)$ and $g_{0}(z)$ and the constants $c_{1}, d_{1}, c_{3 / 2}$ and $d_{3 / 2}$ in $\dot{X}$ and $\dot{\Gamma}$, as we will derive below.

Inserting (44) into (43), we see that the dominant terms are of order $t^{1 / 2}$. Upon collecting these, we get

$$
\left[z \frac{d^{2}}{d z^{2}}+\left(\frac{1}{2}-z\right) \frac{d}{d z}-1-\lambda^{*} c_{1}+i\left(d_{1}+q^{*} c_{1}\right)\right] g_{-1 / 2}=0
$$

This homogeneous equation is an example of Kummer's equation [28]

$$
\hat{T}[a, b] g \equiv\left[z \frac{d^{2}}{d z^{2}}+(b-z) \frac{d}{d z}-a\right] g=0,
$$

whose general solution is a superposition of the two confluent hypergeometric functions

$$
M(a, b, z) \text { and } z^{1-b} M(1+a-b, 2-b, z) .
$$


These functions are defined through the series

$$
M(a, b, z)=\sum_{n=0}^{\infty} \frac{(a)_{n} z^{n}}{(b)_{n} n !}
$$

where

$$
(a)_{n}=a(a+1) \ldots(a+n-1)=\frac{\Gamma(a+n)}{\Gamma(a)}, \quad(a)_{0}=1 .
$$

The asymptotic large- $z$ behavior of the functions $M$ for positive $b$ is

$$
M(a, b, z) \stackrel{z \rightarrow \infty}{\simeq} \begin{cases}\frac{\Gamma(b)}{\Gamma(a)} z^{a-b} & \text { for } a \neq 0,-1,-2,-3, \cdots, \\ \frac{(a)_{|a|} z^{|a|}}{(b)_{|a|}(|a|) !} & \text { for } a=0,-1,-2,-3, \cdots,\end{cases}
$$

Let us return to Eq. (A.5) for $g_{-1 / 2}(z)$. The boundary condition (A.3) implies

$$
g_{-1 / 2}(z)=2 \alpha \sqrt{\mathcal{D} z}+O\left(z^{3 / 2}\right)
$$

Since $M(a, b, z=0)=1$, a contribution of the solution $M(a, b, z)$ is excluded through (A.11), and $g_{-1 / 2}(z)$ has to be proportional to $z^{1-b} M(1+a-b, 2-b, z)$. With boundary condition (A.3), we therefore get

$$
g_{-1 / 2}(z)=2 \alpha \sqrt{\mathcal{D} z} M\left(\frac{3}{2}+\lambda^{*} c_{1}-i\left(d_{1}+q^{*} c_{1}\right), \frac{3}{2}, z\right) .
$$

Furthermore, (A.10) shows that the Kummer functions $M(a, b, z)$ diverge as $e^{z}$ when the coefficient $a$ is not zero or a negative integer, while they are simple polynomials when $a$ is zero or a negative integer since then the coefficients $(a)_{n}$ vanish for $n \geq 1-a$. An exponential divergence of $g$ is not allowed according to the second boundary condition (A.4); this fixes

$$
1+a-b=\frac{3}{2}+\lambda^{*} c_{1}-i\left(d_{1}+q^{*} c_{1}\right)=0,-1,-2, \ldots .
$$

For a detailed discussion of the solutions with $1+a-b=-1,-2, \ldots$, we refer to [6]: essentially, these solutions are dynamically not relevant since they will always be overrun by the solution with $1+a-b=0$. As both $c_{1}$ and $d_{1}$ are real, (A.13) with $1+a-b=0$ implies

$$
c_{1}=-\frac{3}{2 \lambda^{*}}, \quad d_{1}=-q^{*} c_{1},
$$


with the corresponding solution

$$
g_{-1 / 2}(z)=2 \alpha \sqrt{\mathcal{D} z}
$$

The terms of order $t^{0}$ obtained by subsituting (44) into (43) are

$$
\begin{aligned}
& \hat{T}\left[\frac{1}{2}+\lambda^{*} c_{1}-i\left(d_{1}+q^{*} c_{1}\right), \frac{1}{2}\right] g_{0}(z)= \\
& {\left[-i k^{*} c_{3 / 2}-c_{1} \frac{\sqrt{z}}{\sqrt{\mathcal{D}}}\left(\partial_{z}-1\right)-i d_{3 / 2}\right] g_{-1 / 2}(z)} \\
& -\frac{\mathcal{D}_{3} \sqrt{z}}{\mathcal{D}^{\frac{3}{2}}}\left[\frac{3}{2}\left(\partial_{z}-1\right)^{2}+z\left(\partial_{z}-1\right)^{3}\right] g_{-1 / 2}(z) \\
& -w \frac{\sqrt{z}}{\sqrt{\mathcal{D}}}\left[\frac{1}{2}-z\left(\partial_{z}-1\right)-1-i k^{*} c_{1}-i d_{1}\right]\left(\partial_{z}-1\right) g_{-1 / 2}(z) .
\end{aligned}
$$

The function $g_{-1 / 2}(z)$ on the right hand side of (A.16) is known from (A.15); likewise $c_{1}$ and $d_{1}$ are known from (A.14). Substitution of these results gives the following inhomogeneous equation for $g_{0}(z)$

$$
\begin{aligned}
\hat{T}\left[-1, \frac{1}{2}\right] g_{0}(z)=2 & \alpha\left[c_{3 / 2} \lambda^{*}-i\left(d_{3 / 2}+q^{*} c_{3 / 2}\right)\right] \sqrt{\mathcal{D} z}+\frac{3 \alpha}{2 \lambda^{*}}(1-2 z) \\
& +2 \alpha \frac{\mathcal{D}_{3}}{\mathcal{D}}\left[z^{2}-3 z+\frac{3}{4}\right]+2 \alpha w\left[z^{2}-3 z+\frac{3}{4}\right] .
\end{aligned}
$$

The general solution of this inhomogeneous equation is a particular solution plus the sum of two independent solutions of the homogeneous equation $\hat{T}\left[-1, \frac{1}{2}\right] g_{0}(z)=0$. The latter can again be written in terms of Kummer functions. It is easy to find particular solutions which reproduce most of the terms on the right by noting that

$$
\begin{aligned}
\hat{T}\left[-1, \frac{1}{2}\right] \sqrt{z} & =\frac{1}{2} \sqrt{z}, & \hat{T}\left[-1, \frac{1}{2}\right] 1 & =1, \\
\hat{T}\left[-1, \frac{1}{2}\right] z & =\frac{1}{2}, & \hat{T}\left[-1, \frac{1}{2}\right] z^{2} & =-z^{2}+3 z .
\end{aligned}
$$

With these terms, we can generate all the terms on the right hand side of (A.16), except for the term linear in $z$. We can generate this term by noting that the function

$$
F_{N}(z)=\sum_{n=N}^{\infty} \frac{(1)_{n-2} z^{n}}{\left(\frac{1}{2}\right)_{n} n !}
$$


is proportional to a truncated Kummer series $M\left(1, \frac{1}{2}, z\right)$ (see below) and solves

$$
\hat{T}\left[-1, \frac{1}{2}\right] F_{N}(z)=\frac{z^{N-1}}{\left(\frac{1}{2}\right)_{N-1}(N-1)}, \quad \text { hence } \hat{T}\left[-1, \frac{1}{2}\right] F_{2}(z)=2 z .
$$

Using all the results (A.7), (A.18) and (A.20), we can write the general solution of (A.17) as

$$
\begin{aligned}
g_{0}(z)=k_{0}(1 & -2 z)+l_{0} \sqrt{z} M\left(-\frac{1}{2}, \frac{3}{2}, z\right) \\
& +4 \alpha\left[c_{3 / 2} \lambda^{*}-i\left(d_{3 / 2}+q^{*} c_{3 / 2}\right)\right] \sqrt{\mathcal{D} z} \\
& +\frac{3 \alpha}{2 \lambda^{*}}\left[1-F_{2}(z)\right]-2 \alpha\left(\frac{\mathcal{D}_{3}}{\mathcal{D}}+w\right)\left[z^{2}-\frac{3}{4}\right] .
\end{aligned}
$$

where we used the fact that $M\left(-1, \frac{1}{2}, z\right)=1-2 z$. The parameters $k_{0}, l_{0}, c_{3 / 2}$ and $d_{3 / 2}$ are again determined by the boundary conditions. First, the boundary condition (A.3) implies for $g_{0}$ that $g_{0}(z)=\beta+\mathcal{O}(z)$. This gives with (A.21)

$$
\begin{aligned}
\beta+\mathcal{O}(z)=[ & \left.k_{0}+\frac{3 \alpha}{2}\left(\frac{1}{\lambda^{*}}+\frac{\mathcal{D}_{3}}{\mathcal{D}}+w\right)\right] \\
& +\left[4 \alpha\left(c_{3 / 2} \lambda^{*}-i\left(d_{3 / 2}+q^{*} c_{3 / 2}\right)\right) \sqrt{\mathcal{D}}+l_{0}\right] \sqrt{z}+\cdots
\end{aligned}
$$

The first term on the right determines the coefficient $k_{0}$ in terms of $\alpha, \beta$ and the other parameters, but this term is not needed in the sequel. The condition that the prefactor of the $\sqrt{z}$ term on the right vanishes gives

$$
\left(c_{3 / 2} \lambda^{*}-i\left(d_{3 / 2}+q^{*} c_{3 / 2}\right)\right) \sqrt{\mathcal{D}}+\frac{l_{0}}{4 \alpha}=0 .
$$

Second, the boundary condition (A.4) imposes also for $g_{0}(z)$, that the function does not diverge exponentially for large $z$. There are two terms in (A.21) which diverge exponentially: the Kummer function $M$, whose asymptotic behavior is given in (A.10), and the function $F_{2}(z)$. It is easy to see that for large $z$, we have

$$
z^{2} \frac{\mathrm{d}^{2} F_{2}(z)}{\mathrm{d} z^{2}} \simeq M\left(1, \frac{1}{2}, z\right) \quad \Longrightarrow \quad F_{2}(z) \simeq \sqrt{\pi} z^{-3 / 2} e^{z}
$$

Therefore the requirement that the two exponentially divergent terms in $g_{0}(z)$ cancel each other, translates into

$$
\frac{l_{0}}{4 \alpha}+\frac{3 \sqrt{\pi}}{2 \lambda^{*}}=0
$$


Upon eliminating $l_{0} / \alpha$ from equations (A.23) and (A.25) we simply get

$$
c_{3 / 2}=\frac{3 \sqrt{\pi}}{2\left(\lambda^{*}\right)^{2}} \operatorname{Re} \frac{1}{\sqrt{\mathcal{D}}}, \quad d_{3 / 2}=-\frac{3 \sqrt{\pi}}{2 \lambda^{*}} \operatorname{Im} \frac{1}{\sqrt{\mathcal{D}}}-q^{*} c_{3 / 2} .
$$

The second contribution to $d_{3 / 2}$ is just the contribution to the phase relaxation which is induced by the relaxation of $v(t)$. Upon substitution of these results in the expansions (32) for $X(t)$ and (34) for $\Gamma(t)$ we get the results (45) and (46).

\section{References}

[1] R. J. Briggs, Electron-Stream Interaction with Plasmas (MIT Press, Cambridge, 1964).

[2] A. N. Bers, Space-Time Evolution of Plasma Instabilities - Absolute and Convective, in: Handbook of Plasma Physics, M. N. Rosenbluth and R. Z. Sagdeev, eds. (North-Holland, Amsterdam, 1983).

[3] L. D. Landau and E. M. Lifshitz, Electrodynamics of Continuous Media, vol. 8 of Course of Theoretical Physics (Pergamon, New York, 1975).

[4] P. Huerre, in: Propagation in Systems far from Equilibrium, J. E. Wesfreid, H. R. Brand, P. Manneville, G. Albinet, and N. Boccara, eds (Springer, New York, 1988).

[5] U. Ebert and W. van Saarloos, Universal algebraic relaxation of fronts propagating into unstable state and implications for moving boundary approximations, Phys. Rev. Lett. 80, 1650 (1998).

[6] U. Ebert and W. van Saarloos, Front propagation into unstable states: universal algebraic convergence towards uniformly pulled fronts, Physica D 146, 1 (2000).

[7] W. van Saarloos, Front propagation into unstable states, Phys. Rep. 386, 29 (2003).

[8] A. N. Stokes, On Two Types of Moving Fronts in Quasilinear Diffusion, Math. Biosciences 31, 307 (1976).

[9] G. C. Paquette, L.-Y. Chen, N. Goldenfeld, and Y. Oono, Structural Stability and Renormalization Group for Propagating Fronts, Phys. Rev. Lett. 72, 76 (1994).

[10] U. Ebert and W. van Saarloos, Breakdown of the standard Perturbation Theory and Moving Boundary Approximation for "Pulled" Fronts, Phys. Rep. 337, 139 (2000).

[11] R. A. Fisher, The wave of advance of advantageous genes, Ann. Eugenics 7, 355 (1937). 
[12] A. Kolmogoroff, I. Petrovsky, and N. Piscounoff, Study of the Diffusion Equation with Growth of the Quantity of Matter and its Application to a Biology Problem, Bulletin de l'université d'état à Moscou, Ser. int., Section A, Vol. 1 (1937).

[13] M. Bramson, Convergence of Solutions of the Kolmogorov equation to traveling waves, Mem. Am. Math. Soc. 44, No. 285 (1983).

[14] U. Ebert, W. van Saarloos and L. A. Peletier, Universal algebraic convergence in time of pulled fronts: the common mechanism for difference-differential and partial differential equations, Euro. Jnl. Appl. Math. 13, 53 (2002).

[15] E. Brunet and B. Derrida, Shift of the velocity of a front due to a cutoff, Phys. Rev. E 56, 2597 (1997).

[16] C. Storm, W. Spruijt, U. Ebert and W. van Saarloos, Universal Algebraic Relaxation of Velocity and Phase in Pulled Fronts generating Periodic or Chaotic States, Phys. Rev. E 61, R6063 (2000).

[17] G. Dee and J. S. Langer, Propagating Pattern Selection, Phys. Rev. Lett. 50, 383 (1983).

[18] W. van Saarloos, Front Propagation into Unstable States II: Linear versus Nonlinear Marginal Stability and Rate of Convergence, Phys. Rev. A 39, 6367 (1989).

[19] P. Collet and J. P. Eckmann, The Stability of Modulated Fronts, Helv. Phys. Acta 60, 969 (1987).

[20] P. Collet and J. P. Eckmann, Instabilities and Fronts in Extended Systems (Princeton University Press, Princeton, 1990).

[21] J.-P. Eckmann and C. E. Wayne, Propagating Fronts and the Center Manifold Theorem, Commun. Math. Phys. 161, 323 (1994).

[22] P. Collet and J. P. Eckmann, A rigorous upper bound on the propagation speed for the Swift-Hohenberg and related equations, J. Stat. Phys. 108, 1107 (2002).

[23] G. Dee and W. van Saarloos, Bistable Systems with Propagating Fronts Leading to Pattern Formation, Phys. Rev. Lett. 60, 2641 (1988).

[24] L. A. Peletier and W. C. Troy, Spatial Patterns: Higher order Models in Physics and Mechanics (Birkäuser, Boston, 2001).

[25] R. van Zon, H. van Beijeren and Ch. Dellaga, Largest Lyapunov exponent for many-particle systems at low densities, Phys. Rev. Lett. 80, 2035 (1998).

[26] K. Nozaki and N. Bekki, Pattern Selection and Spatio-temporal Transition to Chaos in the Ginzburg-Landau equation, Phys. Rev. Lett. 51, 2171 (1983).

[27] W. van Saarloos and P. C. Hohenberg, Fronts, Pulses, Sources and Sinks in Generalized Complex Ginzburg-Landau Equations, Physica D 56, 303 (1992) [Errata: Physica D 61, 209 (1993)].

[28] M. Abramowitz and I. S. Stegun (Eds.), Handbook of Mathematical Functions (Dover, New York, 1972). 